\title{
Improving Cattle Market Syndromic Surveillance Through Electronic Data Capture
}

\author{
Leah Estberg ${ }^{1}$, Randy Munger ${ }^{1}$, Cynthia Zepeda ${ }^{1}$, Judy Akkina* ${ }^{\star 1}$, Susan Rollo ${ }^{2}$ and \\ Andy Schwartz ${ }^{2}$
}

${ }^{1}$ USDA-APHIS-VS, Fort Collins, CO, USA; ${ }^{2}$ Texas Animal Health Commission, Austin, TX, USA

\section{Objective}

Implement a mobile technology platform to capture and transmit syndromic cattle data collected at Texas market sales.

\section{Introduction}

An active syndromic surveillance system was designed to collect cattle health information from a sample of Texas cattle market sales. Texas Animal Health Commission livestock inspectors record the total number of animals observed along with the total number displaying clinical signs of interest grouped into body system categories (e.g. respiratory, neurologic, etc.). Inspection reports are submitted to the United States Department of Agriculture Veterinary Services (VS) Risk Identification Team for monitoring.

\section{Methods}

The pilot project started in 2012 with paper-based data collection forms to both 1) gain trust from the inspector supervisors and 2) evaluate the value of the system with minimal early investment. The data collected at each sale on paper-based forms were later entered into spreadsheets at the office. These sale inspection reports were then submitted to the inspector's supervisor for review prior to forwarding by email to VS. VS staff aggregated data from each spreadsheet in to a centralized database and conducted weekly monitoring.

Recently, a new reporting system was developed at VS to enable collection and transmission of the data on mobile devices running an Android operating system capable of transmitting data to VS via a Wi-Fi connection. The new system was deployed March 2016 following in-person training, release of a user guide document, and a month of user testing.

\section{Results}

Between March 2014 and June 2016 a total of 1,330 sale inspection reports from 16 markets were submitted by spreadsheet an average 11 days following the sale (range: 1 day through 141 days following the sale). These reports were tracked for data quality issues that required manual intervention. It was discovered that $64(4.8 \%)$ of the reports required correction. The most common types of data quality issues were market sale date not provided, market alias ID not provided, report submitted more than once, and report not submitted as an Excel file but as an image, such as a pdf file.

Between March and June 2016 a total of 160 sale inspection reports from 16 markets were submitted using mobile devices an average 7 days following the sale (range: same day through 47 days following the sale). All data submitted could be directly imported into the centralized database and processed as needed for monitoring without any data correction required.

Some challenges encountered with deploying the mobile technology system included addressing the VS Information Technology security requirements for establishing user accounts and implementing direct data upload into VS systems. Additionally, Wi-Fi connectivity can be difficult in some remote areas.

Some advantages to using the mobile technology included having the option to download and run the application on most mobile devices running the Android operating system. There was an improvement in data reporting timeliness of 4 days on average, and the range substantially narrowed. There was also time savings for inspectors who no longer needed to transfer hard copy data to a spreadsheet, and for VS personnel who no longer needed to aggregate data from individual spreadsheets. Improvements in data quality included the ability to directly report that sales were canceled or not attended; the ability to provide comments at various levels of detail related to the sale, the pen of animals observed, or specific signs observed; and the requirement to supply essential data elements such as sale date and market ID

\section{Conclusions}

The conversion from a paper and spreadsheet-based sale inspection report to a mobile technology platform resulted in significant time savings and data quality improvements that appeared to justify the system development and deployment costs and challenges. These benefits support potential expansion of the system.

\section{Keywords}

Animal health monitoring; Mobile technology; Electronic data collection

\section{Acknowledgments}

This work would not have been successful without the effort from multiple individuals at the Texas Animal Health Commission. This work was also supported by USDA VS with funding and personnel resources.

\footnotetext{
*Judy Akkina

E-mail: judy.e.akkina@aphis.usda.gov
} 